\title{
Magnetic hyperthermia enhance the treatment efficacy of peri-implant osteomyelitis
}

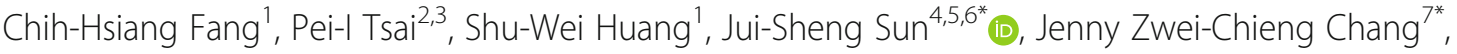 \\ Hsin-Hsin Shen ${ }^{8}$, San-Yuan Chen ${ }^{2}$, Feng Huei Lin', Lih-Tao Hsu ${ }^{9}$ and Yen-Chun Chen ${ }^{8}$
}

\begin{abstract}
Background: When bacteria colony persist within a biofilm, suitable drugs are not yet available for the eradication of biofilm-producing bacteria. The aim of this study is to study the effect of magnetic nano-particles-induced hyperthermia on destroying biofilm and promoting bactericidal effects of antibiotics in the treatment of osteomyelitis.

Methods: Sixty 12-weeks-old male Wistar rats were used. A metallic 18G needle was implanted into the bone marrow cavity of distal femur after the injection of Methicillin-sensitive Staphylococcus aureus (MSSA). All animals were divided into 5 different treatment modalities. The microbiological evaluation, scanning electron microscope examination, radiographic examination and then micro- $C T$ evaluation of peri-implant bone resorption were analyzed.

Results: The pathomorphological characteristics of biofilm formation were completed after 40-days induction of osteomyelitis. The inserted implants can be heated upto $75^{\circ} \mathrm{C}$ by magnetic heating without any significant thermal damage on the surrounding tissue. We also demonstrated that systemic administration of vancomycin [VC (i.m.)] could not eradicate the bacteria; but, local administration of vancomycin into the femoral canal and the presence of magnetic nanoparticles hyperthermia did enhance the eradication of bacteria in a biofilm-based colony. In these two groups, the percent bone volume (BV/TV: \%) was significantly higher than that of the positive control.

Conclusions: For the treatment of chronic osteomyelitis, we developed a new modality to improve antibiotic efficacy; the protection effect of biofilms on bacteria could be destroyed by magnetic nanoparticles-induced hyperthermia and therapeutic effect of systemic antibiotics could be enhanced.
\end{abstract}

Keywords: Peri-implant osteomyelitis, Magnetic nanoparticle, Hyperthermia, Biofilm

\section{Background}

Infection of bone and soft tissue is one of the biggest problems in orthopedic daily practice, which mostly leads to progressive inflammation and even bone destruction [1]. Osteomyelitis is the infection and inflammation of bone and bone marrow tissue, usually begins as an acute infection, but it may evolve into a chronic condition. It can be subdivided into hematogenous or posttraumatic spread of bacteria, whereas the incidence of posttraumatic osteomyelitis is around $80 \%$ [2]. The treatment of chronic

\footnotetext{
* Correspondence: drjssun@gmail.com; jennyzc@ms3.hinet.net

${ }^{4}$ Department of Orthopedic Surgery, College of Medicine, National Taiwan

University, No. 1, Ren-Ai Rd, Taipei 10051, Taiwan, Republic of China

${ }^{7}$ School of Dentistry, College of Medicine, National Taiwan University, No 1

Chang-Te Street, Taipei 10048, Taiwan

Full list of author information is available at the end of the article
}

osteomyelitis is a challenging problem, since germs with variable drug sensitivities exist. The most common pathogens are coagulase negative staphylococci and Staphylococcus aureus [3]. There is still no evidencebased guidance on the treatment of chronic osteomyelitis and there is no single-treatment regimen or surgical procedure that is appropriate for all patients [2]. Besides the use of antibiotics, sufficient debridement seems to be the most important therapy for osteomyelitis [2].

In the presence of necrotic bone, a biofilm can develop and reduce the efficacy of antibiotic therapy by $10^{3}$ folds [4]. Bacteria may persist in a biofilm-based colony or be intra-cellularly concealed within osteoblasts, these characteristics render bacteria a greater resistance to the host's immune response and antibiotic therapy [5]. Biofilm is 
any group of microorganisms in which cells stick to each other and adhere to a surface. These adherent cells are frequently embedded within a self-produced matrix of extracellular polymeric substance (EPS) [6]. Suitable drugs are not yet available for the eradication of such biofilmproducing bacteria.

Iron is an essential but potentially hazardous biometal. Mammalian cells require sufficient amounts of iron to satisfy metabolic needs or to accomplish specialized functions. Iron is an essential trace nutrient required for the active sites of many electron transfer and oxygen transport enzymes. In contrast, iron is also a catalyst for reactive oxygen species (ROS). Organisms must acquire iron but also must protect against oxidative damage. Ferritin is a naturally designed nanomachine for disposing cellular irons. Ferritin sequesters iron and maintains it in a biologically safe form. Ferritin also releases stored metals in response to cell needs. Thus, ferritin plays important roles in detoxification and reservation, and is vital for maintaining iron homeostasis in living cells. Ferritin genes are ubiquitously present among bacteria, plants, and animals, suggesting that they are essential genes for most organisms [7-9]. The iron oxide particle size of these agents varies widely, and influences their physicochemical and pharmacokinetic properties, and thus their clinical applications. Iron oxide nanocrystals (IONPs) are superparamagnetic. Many IONPs, including superparamagnetic iron oxides (SPIOs), are encapsulated by hydrophilic polymers to improve the stability and solubility. SPIOs have been used in clinical applications as a contrast agent for magnetic resonance imaging (MRI) $[10,11]$. IONPs can be safely administered in the body because they do not raise concerns of heavy metal uptake and accumulation.

The healing effect of heat has been well-known since ancient times, it have been used to cure cancer [12] and infection [13]. The heating of biological tissue can be achieved by several physical mechanisms including microwave irradiation [14, 15], Ohmic heating [16], optical laser irradiation [17] or water bath heating $[18,19]$. With these heating methods, it remains a challenge to control the spatial extent of heating in tissue. Magnetic particles hyperthermia improves the precision of heating by embedding the heating source into tissue and heating it using an external alternating magnetic field [20]. Many clinical trials have demonstrated the efficacy and safety of magnetic hyperthermia for prostate cancer and gliomas; patients can tolerate this therapy without discomfort or serious side effects [21-23]. However, there is no research for magnetic hyperthermia in the treatment of osteomyelitis. The aim of this study is to study the heating effect of magnetic nanoparticles-induced hyperthermia on destroying biofilm and promoting antibiotic efficacy for the treatment of peri-implant osteomyelitis.

\section{Mthods}

\section{Synthesis of $\mathrm{Fe}_{3} \mathrm{O}_{4}$ magnetic nanoparticles}

All chemicals, including 1,2-tetradecanediol, dibenzyl ether solution, oleic acid $\left(\mathrm{C}_{17} \mathrm{H}_{33} \mathrm{COOH}\right.$, technical grade $\left.90 \%\right)$, oleylamine $\left(\mathrm{C}_{18} \mathrm{H}_{35} \mathrm{NH}_{2}\right.$, technical grade $\left.70 \%\right)$, Iron(III) acetylacetonate were obtained from $\left(\mathrm{Fe}(\mathrm{acac})_{3}\right.$, SigmaAldrich, Inc., St. Louis, MO, USA). $\mathrm{Fe}_{3} \mathrm{O}_{4}$ magnetic nanoparticles were synthesized according to the following procedure. First, 2.0 mmole of $\mathrm{Fe}(\mathrm{acac})_{3}, 10 \mathrm{mmole}$ 1,2-tetradecanediol solution was added into $15 \mathrm{~mL}$ of dibenzyl ether solution, mixed with stabilizer and dispersant [containing 6 mmole oleic acid $\left(\mathrm{C}_{17} \mathrm{H}_{33} \mathrm{COOH}\right)$ and 6 mmole oleylamine $\left.\left(\mathrm{C}_{18} \mathrm{H}_{35} \mathrm{NH}_{2}\right)\right]$, stirring for $30 \mathrm{~min}$ at $130{ }^{\circ} \mathrm{C}$ and then then under a blanket of nitrogen, heated to reflux $\left(290{ }^{\circ} \mathrm{C}\right)$ for $1 \mathrm{~h}$. The dibenzyl ether which containing $0.03 \mathrm{M} \mathrm{Fe}^{3+}$ acetylacetonate mixture solution was added drop-wise under vigorous mechanical stirring $(2000 \mathrm{rpm})$ for $30 \mathrm{~min}$. The color of the suspension turned to black immediately. The suspension was cooled to room temperature by removing the heat source, ethanol $(40 \mathrm{ml})$ was added to the mixture and a black material was precipitated and separated via centrifugation. The black product was dissolved in hexane in the presence of oleic acid $(\sim 0.05 \mathrm{~mL})$ and oleyl amine $(\sim 0.05 \mathrm{~mL})$. Centrifugation $(6000 \mathrm{rpm}, 10 \mathrm{~min})$ was applied to remove any undispersed residue (almost none). The product, $6 \mathrm{~nm} \mathrm{Fe}_{3} \mathrm{O}_{4}$ nanoparticles, was then precipitated with ethanol, centrifuged (6000 rpm, $10 \mathrm{~min})$ to remove the solvent and redispersed into hexane. The precipitate was washed three times with doubly distilled water and then several times with ethanol. The supernatant solution was removed from the precipitate after decantation. Finally, the $6 \mathrm{~nm} \mathrm{Fe}_{3} \mathrm{O}_{4}$ magnetic nanoparticles were dispersed with small amount of oleic acid $(\sim 0.05 \mathrm{~mL})$ and oleylamine $(\sim 0.05 \mathrm{~mL})$ and stored with hexane in a stoppered bottle for further use. The size and morphologies of products were characterized by transmission electron microscopy (TEM) of HITACHI TEM H-7500 microscope (HITACHI, Japan) with 200 mesh carbon film coated copper grid. The crystal structures of samples were recorded using a Rigaku X-ray powder diffractometer (Geigerflex; Tokyo, Japan).

\section{Alternating magnetic field (AMF) generator}

An AMF was generated by a vertical coil with an inner diameter of $6.5 \mathrm{~cm}$ (Coil $1.5 \mathrm{~A}, 30$ turns), driven by a transistor inverter (HOT SHOT; Ameritherm, New York, USA) operated at a frequency of $100 \mathrm{kHz}$ and electric current (EC) $250 \mathrm{~A}$ which is capable of generating a 20G magnetic field. Thermal images were taken using a thermograph (infrared thermal imaging camera InfReC R300SR; Nippon Avionics, Tokyo, Japan). Temperature was also measured by using a thermograph. 
The magnetic properties of $\mathrm{Fe}_{3} \mathrm{O}_{4}$ nanoparticles were analyzed by superconducting quantum interference device (SQUID, MPMS-XL7, Quantum Design, USA) at temperature 2 and $300 \mathrm{~K}$ with an applied field between -20 and $20 \mathrm{kOe}$; moreover, zero field cooling (ZFC) and field cooling (FC) measurement were carried out at 500 Oe to determine the blocking temperature $\left(T_{b}\right)$. The magnetic properties analyses were used to determine the blocking temperature $(\mathrm{Tb})$, the temperature above which the magnetic property will change from ferrimagnetism to superparamagnetism, and magnetization curve which showed the saturation magnetization value for examining the feasibility of temperature increase within the AMF.

\section{In vitro hyperthermia effect}

To evaluate the heating effect in vitro, the magnetic nanoparticles were placed in the bottom of a $1 \mathrm{~mL}$ eppendorf tube filled with $0.5 \mathrm{~mL}$ PBS. Each loaded eppendorf tube was set in the center of a two-turning copper coil $(25 \mathrm{~mm}$ in diameter) in an alternating magnetic field (AMF, Power cube 64/900, 750-1150 kHz, Ceia Co., Arezzo, Italy). Heat generation from the samples was determined by measuring the temperature change of PBS every $10 \mathrm{~s}$ with an optical fiber probe (STF-1 m) connected with the fluoroptic thermometer (I652, Luxtron, USA). The in vitro hyperthermia effect of the samples were carried out in the AMF as mentioned above and the eppendorf tubes were filled with $0.5 \mathrm{~mL}$ FBS. The heating process was performed for $30 \mathrm{~s}$ and the temperature was maintained at 74-75 ${ }^{\circ} \mathrm{C}$ by automatically switching an on/off device of the AMF.

\section{Animal model and surgical procedure}

The experimental protocol was approved by the Institutional Animal Care and Use Committee of Medical College, National Taiwan University (Taipei, Taiwan). Sixty male 12 weeks-old Wistar rats $(300 \pm 20$ g were purchased from the Laboratory Animal Center, Medical College, National Taiwan University (Taipei, Taiwan) and acclimated under standard laboratory conditions at $22 \pm 2{ }^{\circ} \mathrm{C}$ and $50 \pm 10 \%$ humidity. Standard rat chow and water were available ad libitum during the acclimation period. Animals were anesthetized by Zoletil $(15-18 \mathrm{mg} / \mathrm{kg})$ and Rompun $(0.05 \mathrm{ml} / \mathrm{kg})$. Surgical interventions were performed under strict sterile conditions. The surgical area was shaved and disinfected with iodine, and a midline knee incision was made, followed by medial parapatellar arthrotomy and lateral patella subluxation to expose the knee joint. In the pilot study (24 rats), the osteomyelitis model was built by implanting metallic $18 \mathrm{G}$ needle into the bone marrow cavity of distal femur after injecting $10^{5} \mathrm{CFU} / 50 \mu \mathrm{l}$ bacterial suspension of Methicillin-sensitive Staphylococcus aureus BCRC10451 (MSSA); while in the sham-operated negative control rats, metallic $18 \mathrm{G}$ needle was implanted without injection of MSSA (Fig. 1.). The strain used in this study, $S$. aureus BCRC10451 (MSSA), was cultivated overnight at $37{ }^{\circ} \mathrm{C}$ in brain heart infusion (BHI) broth (Sigma-Aldrich, Inc., St. Louis, MO, USA). The arthrotomy was closed using non-resorbable sutures in an interrupted pattern and then the skin was closed with resorbable suture. Intramuscular injection of cefazolie $(20 \mathrm{mg} / \mathrm{kg}$, Purzer Pharmaceutical Co., Ltd., Taipei, Taiwan) was used for postoperative prophylaxis. The formation of biofilm were confirmed by histological staining and scanning electron microscopy. In the pilot study, the rats were randomized to either 10,20 , 30 or 40 days endpoints to check the progression of histopathological change of osteomyelitis. Since the biofilm dormation was quite clear at 20 days after MSSA injection, we selected this time point for the following experiment to evaluate treatment efficacy of hyperthermia.

The evaluation for the treatment efficacy of magnetic hyperthermia, different treatment modalities (total volume $0.5 \mathrm{~cm}^{3}$ ) was implanted into the bone marrow cavity of distal femur by a metallic $23 \mathrm{G}$ needle. All of the 30 animals were divided into 5 groups (Fig. 2): Group I: osteomyelitis positive control without treatment; Group II: osteomyelitis treated with intramuscular injection of vancomycin [VC $(20 \mathrm{mg} / \mathrm{kg}$, i.m., Sandoz Inc., Princeton, NJ, USA)]; Group III: osteomyelitis treated with both intramuscular and femur cavity injection of vancomycin [VC (20 mg/kg, i.m.) + VC (20 mg/ kg, f.c)]; Group IV: osteomyelitis treated with both intramuscular injection of vancomycin and magnetic particles $[\mathrm{VC}(20 \mathrm{mg} / \mathrm{kg}$, i.m.) + M]; Group V: osteomyelitis treated with intramuscular injection of vancomycin and magnetic particles hyperthermia [VC(20 mg/kg, i.m.) + M + IOHA]. Animals from each group were sacrificed by overdose of pentobarbital at 40 days after surgery.

\section{Microbiological evaluation}

Bacteriology swabs from the subcutaneous tissue and the implant site were obtained from all operation sites at the time of sacrifice. Swabs were moistened with one drop $(20 \mu \mathrm{l})$ of sterile PBS, evenly streaked onto agar plate. The samples were incubated for $48 \mathrm{~h}$ at the $37{ }^{\circ} \mathrm{C}$ under anaerobic conditions. After 48 h' incubation, the media were analyzed by conventional bacteriological techniques.

\section{Scanning electron microscope examination and histological staining}

The morphology of biofilm was observed by scanning electron microscopy (SEM; Topcon ABT-60, Tokyo, Japan). Briefly, the specimens were fixed with $4 \%$ paraformaldehyde (PFA) for $2 \mathrm{~h}$ and $2 \%$ osmium tetroxide $\left(\mathrm{OsO}_{4}\right)$ solution for $1 \mathrm{~h}$, dehydrated in a graded series of ethanol, dried by critical-point drying (CPD) method, 

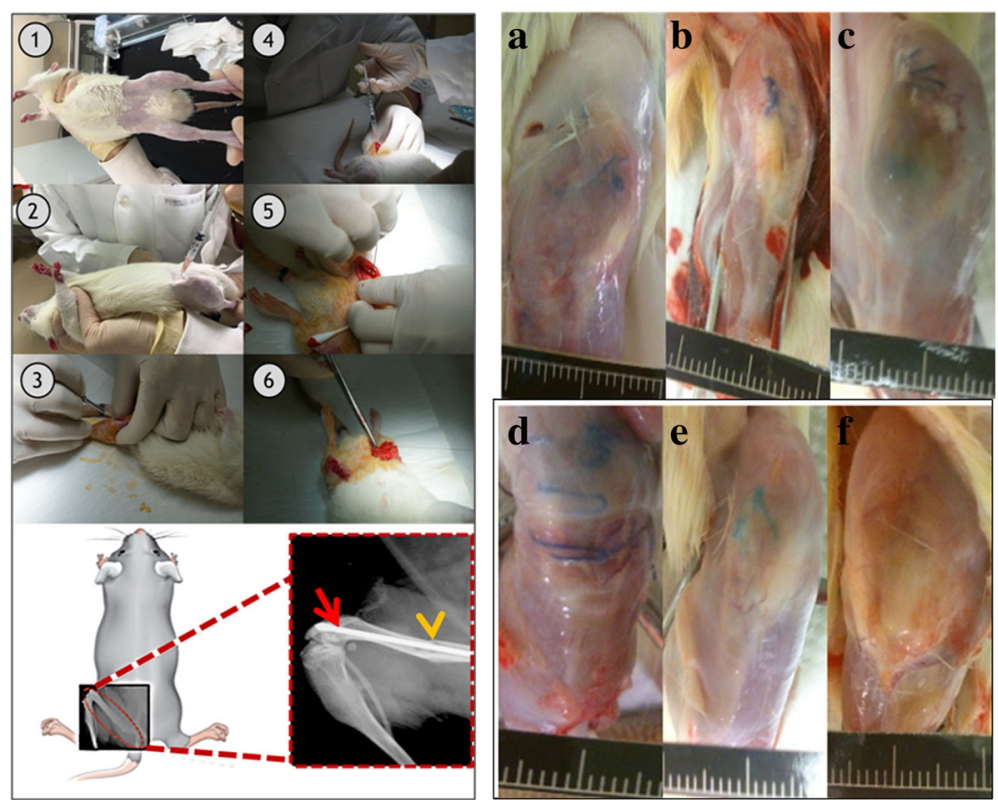

Fig. 1 Sequence of surgical procedures and X-ray image and Animal models for induction of osteomyelitis. Left: Sequence of surgical procedures and X-ray image. Osteomyelitis animal models used $18 \mathrm{G}$ needle as implant and injecting methicillin-sensitive Staphylococcus aureus suspension into bone marrow canal as an infection model. The X-ray image showed that needle completely inserted into femur of rats. Right: Animal models for induction of osteomyelitis. a Uninfected group (left leg); (b) Uninfected group (right leg); (c) Osteomyelitis group (left leg): 10 days after induction of infection; (d) Osteomyelitis group (right leg): 10 days after induction of infection; (e) Osteomyelitis group (left leg): 40 days after induction of infection; (f) Osteomyelitis group (right leg): 40 days after induction of infection

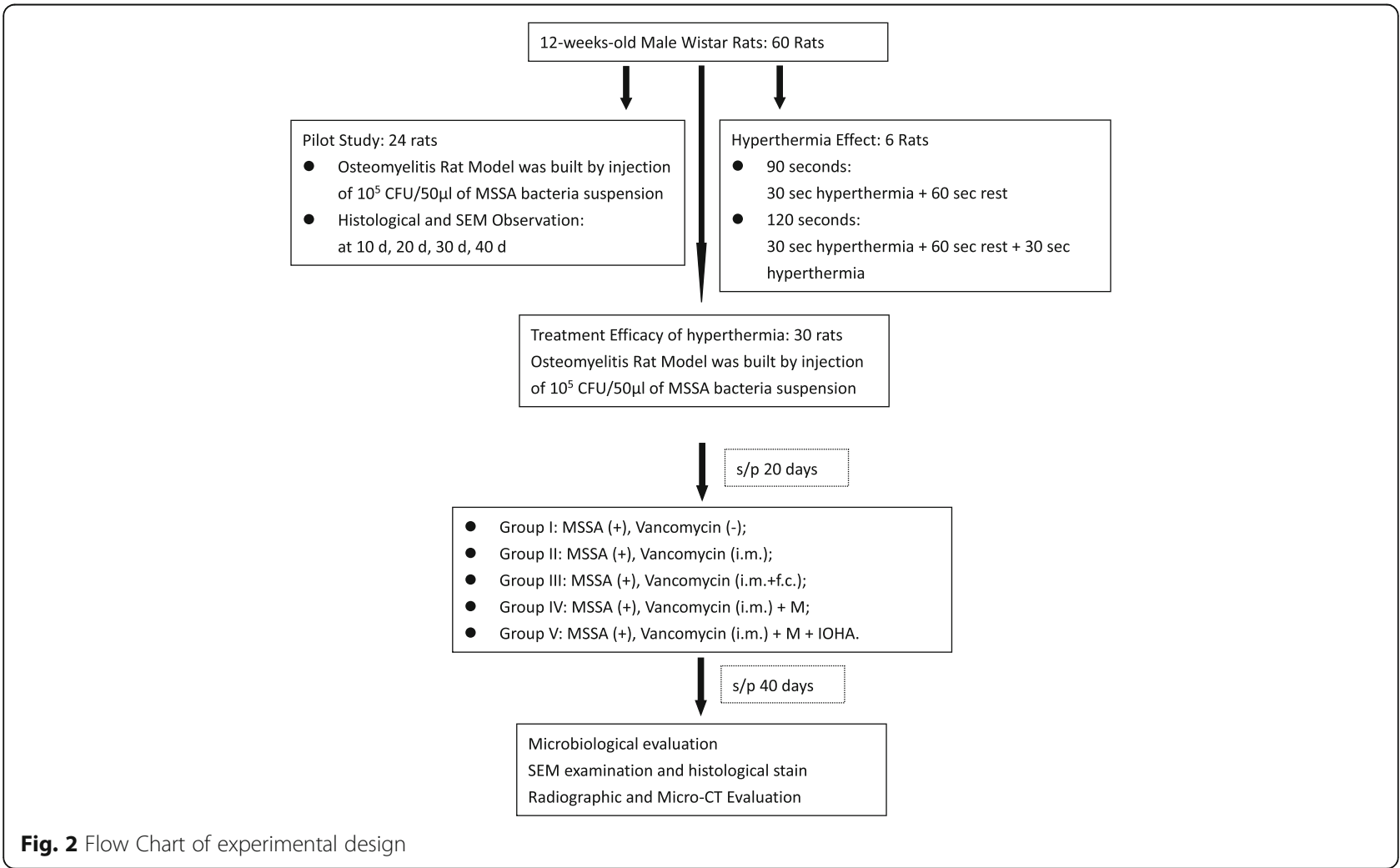

Fig. 2 Flow Chart of experimental design 
and sputter-coated with gold to a thickness film before observation.

For hematoxylin and eosin (HE) staining, randomly chosen femur from the osteomyelitis group was fixed in $5 \%$ formaldehyde for two days, decalcified by $5 \%$ nitric acid solution, and then embedded with paraffin. The tissues were cut and coronally sliced into $7 \mu \mathrm{m}$ sections until use. Slices were stained with haematoxylin/eosin. Congo red staining and gram's staining were also performed using the same sliced tissues. Six visual fields from each section were randomly selected and observed under light microscopy.

\section{Radiographic examination and micro-CT (computed tomography) evaluation}

For radiographic evaluation, the soft tissue attached to the femur was remove, the rat femurs were examined by X-rays (Siemens, Germany). After radiologic examination, implants were removed and then assessed three dimensionally using Micro-CT (SkyScan 1176; Bruker Micro-CT, Kontich, Belgium) [24]. Datasets were reconstructed using CTvox 2.4 software for fast volumetric reconstruction, 2D/3D quantitative analysis and realistic 3D visualization. The tissue volume $\left(\mathrm{TV}: \mathrm{mm}^{3}\right)$, bone volume $\left(\mathrm{BV}: \mathrm{mm}^{3}\right)$ were recorded and then percent bone volume (BV/TV: \%) was analyzed.

\section{Statistical analysis}

All results were presented as means \pm standard deviation (SD). Statistical analysis was performed for all the quantitative results using ANOVA test, the post hoc analysis used was Tukey's honestly significant difference. The overall analysis was performed by the IBM Statistics SPSS 16.0 and the statistical significance in each test was set at $p<0.05$.

\section{Results}

Transmission electron microscope (TEM) examination of $\mathrm{Fe}_{3} \mathrm{O}_{4}$ magnetic nanoparticles was illustrated in the Fig. 3. In the presence of small amounts of stabilizer and dispersant, hydrophilic and stable $\mathrm{Fe}_{3} \mathrm{O}_{4}$ nanoparticles were synthesized (Fig. 3a); the formation of $\mathrm{F}_{3} \mathrm{O}_{4}$ nanoparticles did not significantly affected by the changes in the injection temperature (Fig. 3b).

The crystal structure of $\mathrm{Fe}_{3} \mathrm{O}_{4}$ nanoparticles was confirmed by XRD spectroscopy. As shown in Fig. 4a, the diffraction peaks at $2 \theta=30.4^{\circ}, 35.5^{\circ}$, and $43.2^{\circ}$ can be well indexed to (220), (311), and (400) planes of the inverse cubic spinel structure of $\mathrm{Fe}_{3} \mathrm{O}_{4}$ (JCPDS card no. 75-1610), respectively; according to the reflection peak positions and relative intensities, which confirms the formation of the $\mathrm{Fe}_{3} \mathrm{O}_{4}$ nanoparticles. The sample formed under a magnetic field is pure $\mathrm{Fe}_{3} \mathrm{O}_{4}$ without any impurity phases $[1,25]$. Figure $4 \mathrm{~b}$ was the hysteresis loops of $\mathrm{Fe}_{3} \mathrm{O}_{4}$ nanoparticles at room temperature. As shown in Fig. $4 \mathrm{~b}$, the hysteresis loops show that saturation magnetizations of $\mathrm{Fe}_{3} \mathrm{O}_{4}$ was $1.0 \mathrm{emu} / \mathrm{g}$ at the field of $1.5 \mathrm{KOe}$, and the absence of coercivity and remanence indicates that the as-prepared $\mathrm{Fe}_{3} \mathrm{O}_{4}$ has superparamagnetic properties above the blocking temperature. The TEM image in Fig. 4c shows that the average size of as-synthesized $\mathrm{Fe}_{3} \mathrm{O}_{4}$ magnetite nanoparticles is ca. $16 \mathrm{~nm}$ with narrow size distribution.
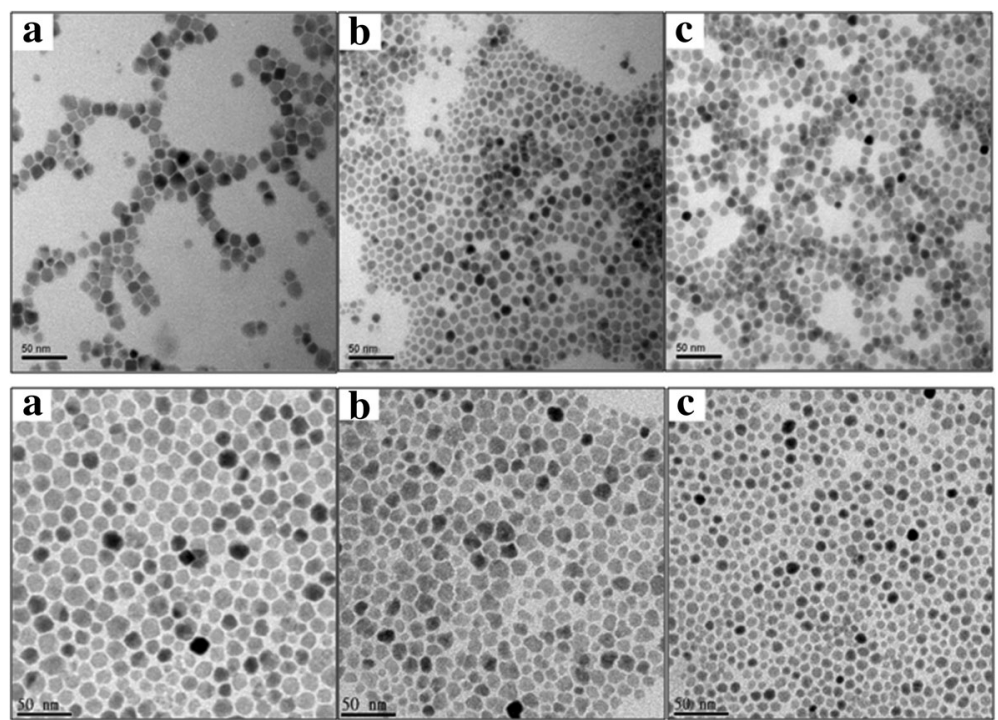

Fig. 3 Transmission Electron Microscope (TEM) examination of $\mathrm{Fe}_{3} \mathrm{O}_{4}$ magnetic nanoparticles. Upper: TEM of $\mathrm{F}_{3} \mathrm{O}_{4}$ Nanoparticles: Effect of different amounts of stabilizer and dispersant. a $80 \mu \mathrm{L}$, b $160 \mu \mathrm{L}$, and c $400 \mu \mathrm{L}$ surfactant. Lower: TEM of $\mathrm{F}_{3} \mathrm{O}_{4}$ Nanoparticles: Effect of different temperature on the formation $\mathrm{F}_{3} \mathrm{O}_{4}$ Nanoparticles. Injection temperature: $\mathbf{a} 260{ }^{\circ} \mathrm{C}$, $\mathbf{b} 230^{\circ} \mathrm{C}$, and $\mathbf{c} 200{ }^{\circ} \mathrm{C}$ 

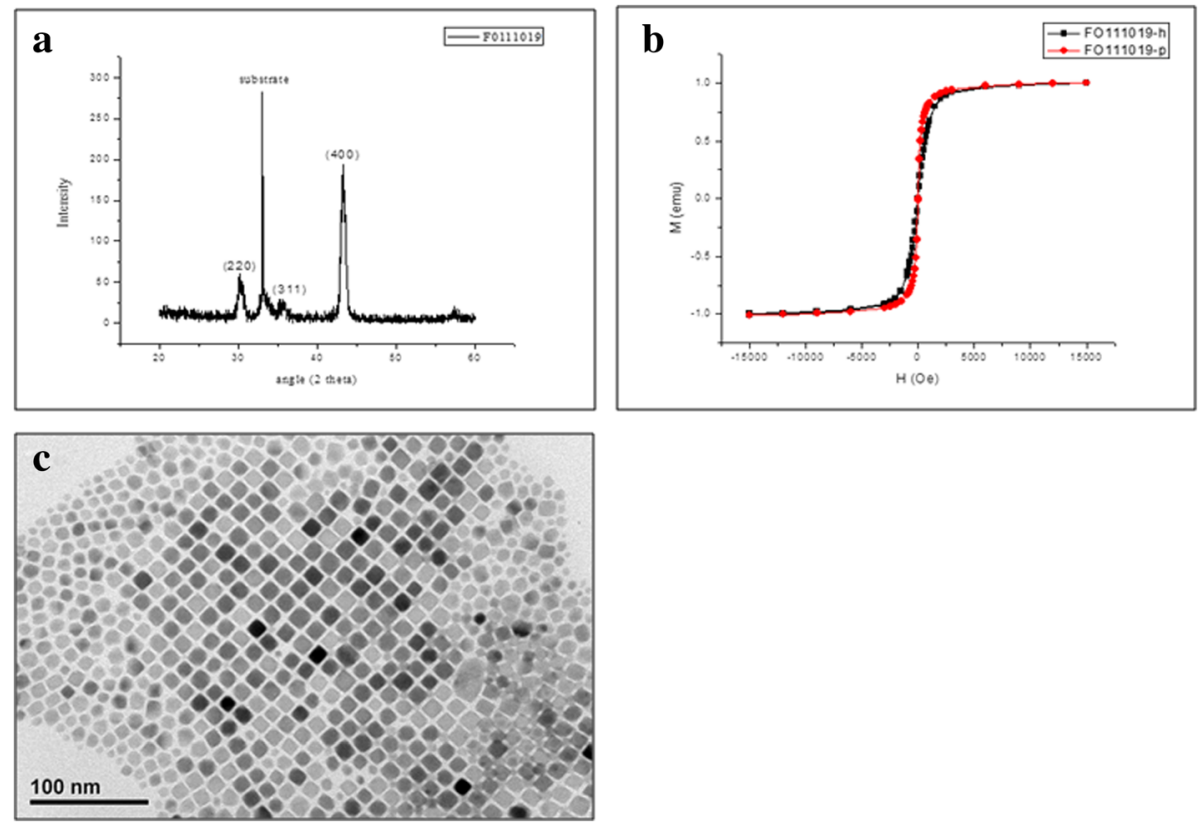

Fig. 4 Characteristics of $\mathrm{Fe}_{3} \mathrm{O}_{4}$ nanoparticles. a The XRD pattern of the $\mathrm{Fe}_{3} \mathrm{O}_{4}$ nanoparticles. $\mathbf{b}$ Room-temperature magnetization curves for FO111019-P and FO111019-h. Magnetic hysteresis curves measured at room temperature for the samples prepared by a hydrothermal process (square: FO111019-h, dot: FO111019-p) showed the super-paramagnetic characteristics. c The crystal structure of $\mathrm{Fe}_{3} \mathrm{O}_{4}$ nanoparticles prepared at the injection temperature of $290^{\circ} \mathrm{C}$ (TEM). The average diameter of particles is $16 \mathrm{~nm}$

Heat production is determined by the magnetic properties of $\mathrm{Fe}_{3} \mathrm{O}_{4}$ nanoparticles, its concentration, and the strength of the AMF. Therefore, we examined the heating effect of AMF on medium containing $\mathrm{Fe}_{3} \mathrm{O}_{4}$ nanoparticles by thermography. The temperature increased timedependently, and we adjusted the required temperature dependent on the concentration of $\mathrm{Fe}_{3} \mathrm{O}_{4}$ nanoparticles and the magnitude of the electric current used to generate AMF to generate a temperature of $75^{\circ} \mathrm{C}$, and we adopted these conditions for the subsequent assays. In this study, the inserted implants can be heated to $75^{\circ} \mathrm{C}$ by magnetic heating (Fig. 5). When the implants were heated to $75{ }^{\circ} \mathrm{C}$ either for $90 \mathrm{~s}$ or $120 \mathrm{~s}$ (each $30 \mathrm{~s}$ heating with a rest for $1 \mathrm{~min}$ ), there were no significant thermal damage noted in these two testing conditions (Fig. 5).

All animals survived after the surgical procedures and no postoperative skin infection or fever was observed. Figure 6 showed the histological, histopathological staining and microbiology assessment from osteomyelitis rats after 40 days infection (Fig. 6. Left upper). In this figure, uninfected group possesses normal trabecular patterns of cancellous bone (upper: Fig. 6 Left upper: A and B); while in the acute suppurative changes of osteomyelitis, there was devitalized lamellar bone with scalloped edges and absence of stainable osteocytes and osteoblasts, edema and granulocytic infiltration of surrounding tissues are obvious (middle: Fig. 6 Left upper: C-E). In the chronic osteomyelitis, there was the irregular fragment of devitalized bone surrounded by dense fibrous tissue heavily infiltrated by plasma cells, lymphocytes, and only a few granulocytes (lower: Fig. 6 Left upper: F-H).

In the histopathological staining, we verified the pathomorphological characteristics of our infected rat femur (Fig. 6 Left lower). As shown here, GRAM-staining revealed the presence of bacteria within the defect site as Gram (+) Staphylococcus aureus bacteria could be detected (Fig. 6 Left lower: B and C). Cango red staining (Fig. 6 Left lower: A) and SEM image (Fig. 6 Left lower: D) showed the biofilm forming completed after 40 days induction of osteomyelitis.

All smears taken from the experimental sites showed characteristic accretion of $S$. aureus infection as depicted in Fig. 3. In the control group, there is few characteristic CFUs on the agar plate (Fig. 6 Right: A). On the other hand, tissue from the affected area, intramedullary liquid from the infected femur and the implants from the infected animals all showed characteristic growth of CFUs on agar plates (Fig. 6 Right: B-D).

After the inoculation of Staphylococcus aureus, tissue necrosis with active inflammation and even biofilm formation were clearly demonstrated in all groups; while, the biofilm formation were found both at the surface of the inserted pin and the inner surface of bony trabeculae. Different treatment modalities did not change the 

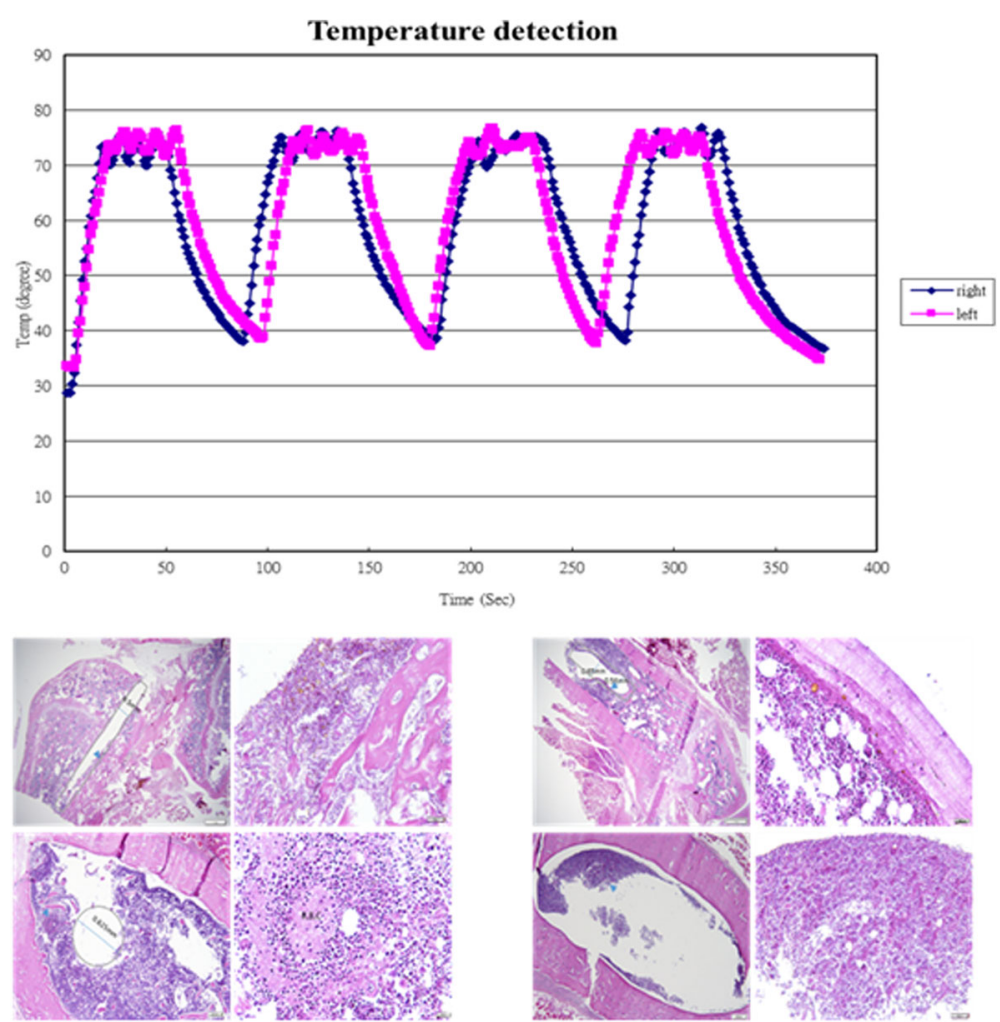

Fig. 5 Tissue damage after thermotherapy ( $\mathrm{H} \& \mathrm{E}$ staining). Left: The needle at $75{ }^{\circ} \mathrm{C}$ for $90 \mathrm{~s}$ by magnetic heating (each $30 \mathrm{~s}$ heating with a rest for $1 \mathrm{~min}$ ), Right: The needle at $75^{\circ} \mathrm{C}$ for $120 \mathrm{~s}$ by magnetic heating (each $30 \mathrm{~s}$ heating with a rest for $1 \mathrm{~min}$ ). There were no significant thermal damage noted in these two testing conditions

histopathological features of the induced osteomyelitis; while massive necrosis of tissue was observed in the group $\mathrm{V}$ which was treated with intramuscular injection of vancomycin and magnetic particles hyperthermia [VC(i.m.) + M + IOHA] (Fig. 7).

In our study, we demonstrated that systemic administration of vancomycin [VC (i.m.)] did not show any effect on eradication of the bacteria when compared with the positive control group; similar event observed in the presence of magnetic nanoparticles [VC (i.m.) + M]. But, local administration of vancomycin into the femoral canal [VC (i.m.) + VC (f.c)] did enhance the eradication of bacteria; similar event was observed in the presence of magnetic nanoparticles when magnetic field was applied [VC (i.m.) + M + IOHA] (Fig. 8).

In the peri-implant osteomyelitis, systemic administration of vancomycin [VC (i.m.)] with/or without the presence of magnetic nanoparticles [VC (i.m.) $+\mathrm{M}$ ] did not affect the percent bone volume; but, when local administration of vancomycin into the femoral canal [VC (i.m.) + VC (f.c)] or magnetic field was applied in the presence of magnetic nanoparticles [VC (i.m.) + M + IOHA]. The percent bone volume (BV/TV) was significantly higher than that of the positive control (Fig. 9).

\section{Discussion}

Currently, there are no evidence-based guidelines in terms of the treatment of chronic osteomyelitis [2]. It is difficult to achieve complete remission, some studies report a failure rates of $20-60 \%$ [26]. The aim of treatment is to improve quality of life through either a curative or a palliative strategy. Curative management strategies usually comprise of a combination of complex surgical procedures and tailored adjuvant antibiotic therapy [27]; on the other hand, palliative treatment strategies are less invasive and typically involve to the use of chronic suppressive antibiotic therapy [28].

Recognizing the importance of the host's physiological status during formulation of a treatment plan, Cierny and Mader revolutionized the approach to chronic osteomyelitis through the publication of their clinical staging system in 1985 (Table 1) [29]. In our model, there is seriously immune response in bone marrow and synovial fluid. Our results exhibit that, after $S$. aureus bacterial inoculation and incubation, a steady infection in femur was generated (Fig. 6 Left upper). According to this classification system, we created a localized type of osteomyelitis in hosts with uncompromised immune systems, corresponding to type IIIA osteomyelitis. In the 

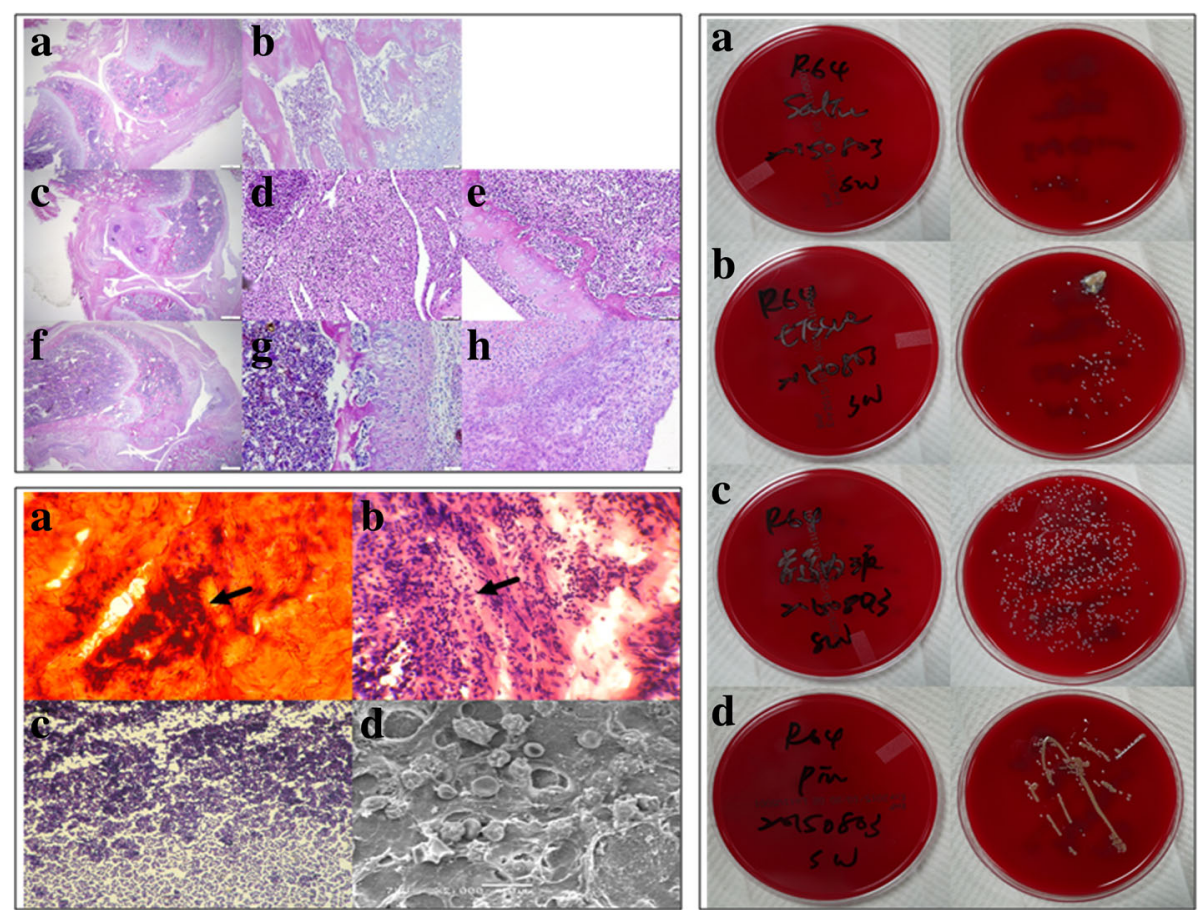

Fig. 6 Histological, histopathological staining and microbiology assessment from osteomyelitis rats after 40 days infection. Left upper: Histological sections with H\&E staining. Upper: Uninfected group, magnification a $40 \times$ and $\mathbf{b} 1000 \times$; Middle: Osteomyelitis 10 days after induction of infection, magnification c $40 \times$, d and $\mathbf{e} 1000 \times$; Lower: Osteomyelitis 40 days after induction of infection, magnification $\mathbf{f} 40 \times, \mathbf{g}$ and $\mathbf{h} 1000 \times$. In this figure, uninfected group possesses normal trabecular patterns of cancellous bone (upper: $\mathbf{a}$ and $\mathbf{b}$ ); while in the acute suppurative changes of osteomyelitis, there is devitalized lamellar bone with scalloped edges and absence of stainable osteocytes and osteoblasts, edema and granulocytic infiltration of surrounding tissues are obvious (middle: $\mathbf{c}-\mathbf{e}$ ). In the chronic osteomyelitis, there is the irregular fragment of devitalized bone surrounded by dense fibrous tissue heavily infiltrated by plasma cells, lymphocytes, and only a few granulocytes (lower: $\mathbf{f}-\mathbf{h}$ ). Left lower: Histopathological staining. a Cango red staining, b H\&E staining, c Gram staining and d SEM morphology, respectively. As shown here, GRAM-staining revealed the presence of bacteria within the defect site as Gram (+) Staphylococcus aureus bacteria could be detected (b and $\mathbf{c})$. Cango red staining (a) and SEM image (d) showed the biofilm forming completed after 40 days induction of osteomyelitis. Magnification of pictures are $1000 \times$. Right: Microbiology assessment from osteomyelitis rats after 40 days infection. Specimens streaked onto agar plate for: a Sterilized PBS as control group; b Tissue from the affected area; $\mathbf{c}$ Intramedullary liquid from the affected femur; $\mathbf{d}$ Implants from the affected animals. All smears taken from the experimental sites showed characteristic accretion of S. aureus infection. In the control group, there is few characteristic CFUs on the agar plate (a). On the other hand, tissue from the affected area, intramedullary liquid from the infected femur and the implants from the infected animals all showed characteristic growth of CFUs on agar plates $(\mathbf{b}-\mathbf{d})$

histopathological staining, we verified the pathomorphological characteristics of our infected rat femur and the biofilm formation was completed after 40 days induction of osteomyelitis (Fig. 6 Left lower), with characteristic accretion of Staphylococcus aureus infection presents at different tissues obtained from the affected area (Fig. 6 Right).

Hyperthermia (HT) is a minimally invasive method for cancer therapy in which the target tissues are annealed to about $42-46{ }^{\circ} \mathrm{C}$. Heat treatment in such a temperature range is cytotoxic for tumor cells because of their disorganized and compact vascular structure that promotes an unfavorable microenvironment inside them. Although HT is a promising approach to cancer therapy, the inevitable technical problem with hyperthermia is the difficulty in heating only the local tumor region to the intended temperature without damaging the surrounding healthy tissue [30]. Magnetic nanoparticles are increasingly used for clinical applications such as drug delivery, magnetic resonance imaging and magnetic fluid hyperthermia. Magnetic nanoparticles (MNPs) have been used for HT treatment in an attempt to overcome this obstacle $[23,31]$. The magnetic nanoparticles have been focus on many research recently because they possess attractive properties which could be potential used in biomedicine, tissue specific targeting, magnetic resonance imaging, defect sensor and environmental remediation, etc. [32-36].

In this study, the inserted implants can be heated to $75{ }^{\circ} \mathrm{C}$ by magnetic heating; while, there were no significant thermal damage noted in this testing condition (Fig. 5) Due to magnetic particles had better dispersion, magnetic nanoparticles (MNPs) heating sources was related to the better distribution of heating intensity in 


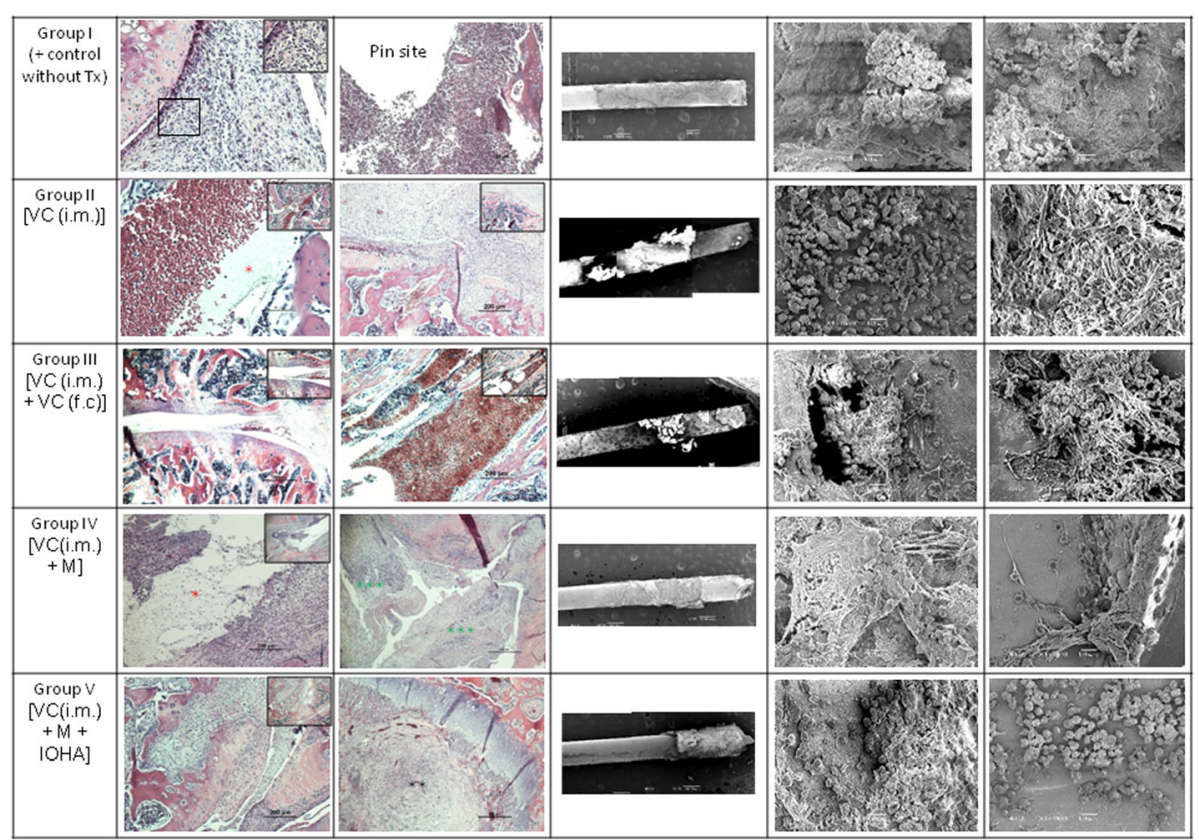

Fig. 7 Histological and scanning electron microscope examination of different treatment modalities for osteomyelitis rats after 40 days infection. After inoculation of Staphylococcus aureus, tissue necrosis with active inflammation and even biofilm formation was clearly demonstrated in all groups; while, the biofilm formation were found both the at the surface of the inserted pin and the inner surface of bony trabeculae. Group I: osteomyelitis positive control without treatment; Group II: osteomyelitis treated with intramuscular injection of vancomycin [VC (i.m.)]; Group III: osteomyelitis treated with both intramuscular and femur cavity injection of vancomycin IVC (i.m.) + VC (f.c)]; Group IV: osteomyelitis treated with both intramuscular injection of vancomycin and magnetic particles [VC(i.m.) + M]; Group V: osteomyelitis treated with intramuscular injection of vancomycin and magnetic particles hyperthermia [VC(i.m.) + M + IOHA]. Animals from each group were sacrificed by overdose of pentobarbital at 40 days after surgery. ${ }^{*}\left(\right.$ red): biofilm-like ECM; ${ }^{* *}$ (black): necrosis with inflammation; ${ }^{* *}($ green): inflammation

tissue [37, 38]. We did observed that after inoculation of Staphylococcus aureus, tissue necrosis with active inflammation and even biofilm formation were clearly demonstrated in all groups; the biofilm formation were found both the at the surface of the inserted pin and the inner surface of bony trabeculae. Different treatment

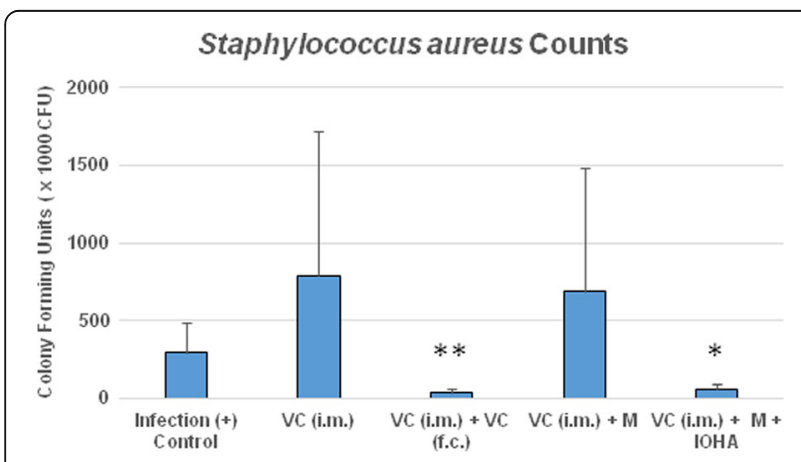

Fig. 8 Colony-forming unit of implants and the affected area under different treatment modalities. Systemic administration of vancomycin [VC (i.m.)] did not show any better effect on eradication of the bacteria; similar event observed in the presence of magnetic nanoparticles IVC (i.m.) + M]. Local administration of vancimycin into the femoral canal [VC (i.m.) + VC (f.c)] did enhance the eradication of bacteria; similar event observed in the presence of magnetic nanoparticles when magnetic field was applied [VC (i.m.) $+\mathrm{M}+\mathrm{IOHA}]$ modalities did not change the histopathological features of the induced osteomyelitis; while massive necrosis of tissue was observed in the group $\mathrm{V}$ which was treated with intramuscular injection of vancomycin and magnetic particles hyperthermia [VC(i.m.) + M + IOHA] (Fig. 7). As shown above, since this parameter did not cause tissue damage, we implied that the induced hyperthermia can enhance the destruction of Staphylococcus aureus.

In this study, rat osteomyelitis model was built by implanting metallic needle into the marrow cavity of distal femur after injecting $10^{8} \mathrm{CFU} / 0.5 \mathrm{ml}$ of bacterial suspension. In this model, intramuscular injection of vancomycin [VC (i.m.)] did not show any effect to treat osteomyelitis. In the group of intramuscular injection of vancomycin with magnetic environment also could not make biofilm collapse and even to enhance bacteria growth $[\mathrm{VC}$ (i.m.) + M]. By raising the temperature of implant, adding magnetic nanoparticles to support hyperthermia that could destroy biofilm and enhance efficacy of vancomycin significantly [VC (i.m.) + M + IOHA] (Fig. 8) . In the peri-implant osteomyelitis, local administration of vancomycin into the femoral canal [VC (i.m.) + VC (f.c)] or magnetic field application in the presence of magnetic nanoparticles [VC (i.m.) + M + IOHA] did prevent the bone loss associated with infection (Fig. 9). 

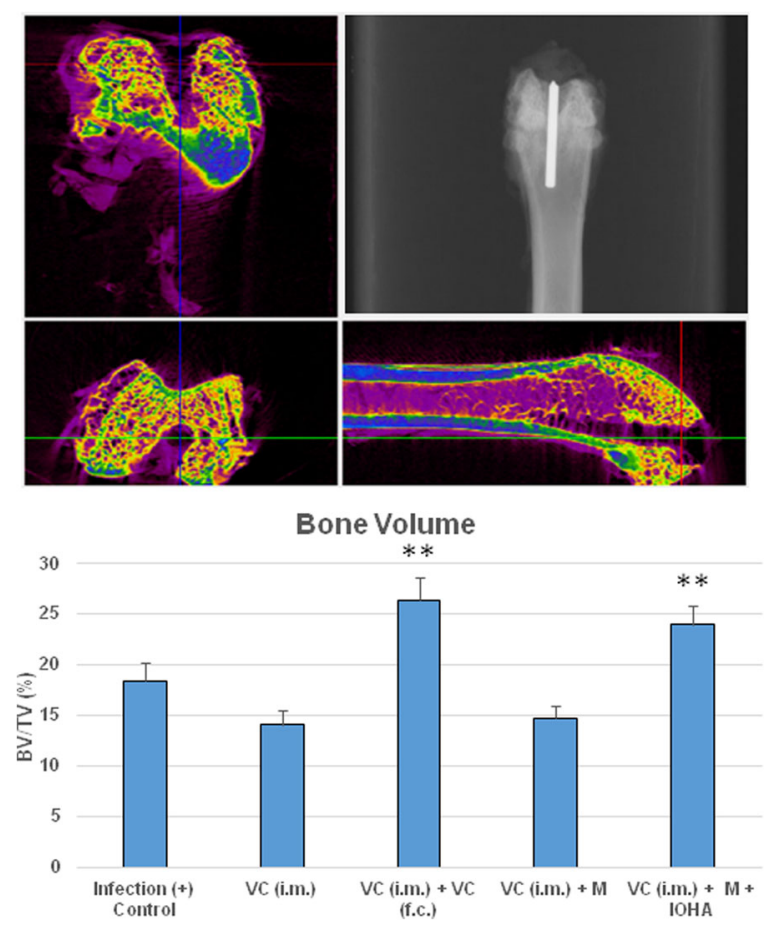

Fig. 9 Change of relative bone volume at the affected area under different treatment modalities. Upper: Micro-CT (Computed Tomography) evaluation and radiographic examination. Lower: Systemic administration of vancomycin [VC (i.m.)] with/or without the presence of magnetic nanoparticles [VC (i.m.) + M] did not affect the bone volume change under peri-implent osteomyelitis condition; but, percent of bone volume (BV/TV) was significantly higher in the group of local administration of vancomycin into the femoral canal [VC (i.m.) + VC (f.c)] and the group when magnetic field was applied in the presence of magnetic nanoparticles [VC (i.m.) $+\mathrm{M}+\mathrm{IOHA}]$

Table 1 Cierny and Mader clinical staging system for adult chronic osteomyelitis $^{\mathbf{2 8}}$

\begin{tabular}{ll}
\hline Anatomic type & \\
|| & Medullary osteomyelitis \\
III & Superficial osteomyelitis \\
IV & Localized osteomyelitis
\end{tabular}

Physiological Class

A Good immune system and delivery

B Compromised locally (BL) or systemically (BS)

C Requires suppressive or no treatment; minimal disability; Clinical Stage treatment worse than disease; not a surgical candidate

Type + Class $=$ Clinical stage
A number of animal models were created in order to study the treatment effects of bacterial infections. Staphylococcus aureus strain for bacterial inoculation was used in almost all of them, because this is the most common pathogen in the setting of osteomyelitis [3, 39]. Furthermore, rats and rabbits were used in most studies because they are big enough to create stable long bone infections without giant efforts; while on the other hand they are small enough to be housed and handled easily [40-43]. Bone and intramedullary bacterial infections are one of the most serious complications of the surgical repair of fractures. Treatment of implant-related chronic osteomyelitis is often difficult and usually consists of implant removal, extensive surgical debridement, and prolonged antibiotic use. To reduce the incidence of implant-related infections, several biomaterial surface treatments with integrated antibiotics, antiseptics, or metal ions have been developed for implants to reduce implant-related infections $[44,45]$. In the current study, we developed a rat model for studies related to improve the treatment of chronic osteomyelitis.

Biofilms are universal, occurring in aquatic and industrial water systems as well as a large number of environments and medical devices relevant for public health. Recent clinical research has implicated biofilms in the exacerbation of wounds [46, 47]. Biofilm formation, which decrease activity of antibiotics, is a crucial step in the pathogenesis of many subacute and chronic bacterial infections, including infection after artificial joints surgery. In this study, we developed a novel antimicrobial thermotherapy platform to treat chronic osteomyelitis. Previous studies always focused on a successful application of hyperthermia in cancer therapy [48, 49]. However, to our knowledge, this is the first in-vivo application of thermotherapy for osteomyelitis. Because of differences in the temperature sensitivity of Staphylococcus aureus compared to cancer cells, transient higher temperature must be safe for realization of this technology as an effective therapeutic adjuvant for infectious pathogens. Our result showed temperature up to $75^{\circ} \mathrm{C}$ is still not causing tissue damage. More important, thermotherapy may be successfully combined with standard-of-care antibiotic treatment for polymicrobial biofilm infections and enhance antibiotic penetration by disrupting the biofilm polysaccharide matrix. This may significantly enhance the efficacy of a given antimicrobial effect and facilitate reduced drug dose, thus providing a valuable tool for the treatment of fragile patients who cannot tolerate high dose systemic antibiotics.

\section{Conclusion}

In this study, we use the needle implantation and injection of methicillin-sensitive Staphylococcus aureus bacterial suspension to create an animal model of osteomyelitis 
which mimic the clinical situation as chronic infection after implantation of artificial materials. Due to biofilms decreased the efficiency of antibiotics, using systemic antibiotic only to treat osteomyelitis did not show therapeutic difference with that of the positive control group. Here, we developed a new treatment modality to improve antibiotic efficacy in the treatment of chronic osteomyelitis. As the smaller size of magnetic naoparticles and their better dispersion in the infected surroundings, nanoparicles-induced hyperthermia can be evenly distributed to the whole surface of biofilm; the biofilms could be destroyed by magnetic nanoparticles hyperthermia and therapeutic effect of systemic antibiotics could be enhanced.

\section{Abbreviations \\ BV/TV: percent bone volume; CPD: critical-point drying; EPS: extracellular polymeric substance; f.c: femur cavity injection; HE staining: hematoxylin and eosin staining; IOHA: magnetic particles hyperthermia; IONPs: iron oxide nanocrystals; M: magnetic particles; Micro-CT: Micro-Computed Tomography; MSSA: methicillin-sensitive Staphylococcus aureus; PFA: para-formaldehyde; ROS: reactive oxygen species; s.c: subcutaneous injection; \\ SPIOs: superparamagnetic iron oxides; TV: tissue volume; VC: vancomycin}

\section{Acknowledgements}

The authors wish to acknowledge thank the staff of the Second Core Lab, Department of Medical Research, National Taiwan University Hospital for technical support during the study.

\section{Funding}

The authors wish to acknowledge Industrial Technology Research Institute, Chutung, Hsinchu, Taiwan, ROC and Research Fund of National Taiwan University Hospital, Taipei, Taiwan, ROC for their funding assistance for this work.

\section{Availability of data and materials}

We had supplied as a supplemental files along with the manuscript and the raw data can be accessed in the additional files.

\section{Authors' contributions}

The substantial contributions of each author was listed below: Chih-Hsiang Fang, Pei-I Tsai, Shu-Wei Huang, Hsin-Hsin Shen, Lih-Tao Hsu, and Yen-Chun Chen:

(1) The conception and design of the study, or acquisition of data, or analysis and interpretation of data; (2) drafting the article or revising it critically for important intellectual content.

San-Yuan Chen, and Feng Huei Lin:

(1) The conception and design of the study, analysis and interpretation of data; (2) drafting the article or revising it critically for important intellectual content; (3) final approval of the version to be submitted.

Jui-Sheng Sun, Jenny, and Zwei-Chieng Chang:

(1) The conception and design of the study, or acquisition of data, or analysis and interpretation of data; (2) drafting the article or revising it critically for important intellectual content; (3) final approval of the version to be submitted.

\section{Ethics approval and consent to participate}

In this manuscripts no human participants, human data or human tissue are included.

The experimental protocol was approved by the Institutional Animal Care and Use Committee of Medical College, National Taiwan University (Taipei, Taiwan).

\section{Consent for publication}

Not applicable.

\section{Competing interests}

The authors declare that there is no benefits in any form have been or will be received from a commercial party related directly or indirectly to the subject of this manuscript.

\section{Publisher's Note}

Springer Nature remains neutral with regard to jurisdictional claims in published maps and institutional affiliations.

\section{Author details}

${ }^{1}$ Institute of Biomedical Engineering, National Taiwan University, Taipei, Taiwan. ${ }^{2}$ Department of Materials Science and Engineering, National Chiao-Tung University, Hsinchu 30010, Taiwan. ${ }^{3}$ Biomedical Technology and Device Research Laboratories, Industrial Technology Research Institute, Chutung, Hsinchu 31040, Taiwan. ${ }^{4}$ Department of Orthopedic Surgery, College of Medicine, National Taiwan University, No. 1, Ren-Ai Rd, Taipei 10051, Taiwan, Republic of China. ${ }^{5}$ Department of Orthopedic Surgery, National Taiwan University Hospital, No.7, Chung-Shan South Rd, Taipei 10002, Taiwan, Republic of China. ${ }^{6}$ Biomimetic Systems Research Center, National Chiao-Tung University, 1001 University Road, Hsinchu 300, Taiwan, Republic of China. 'School of Dentistry, College of Medicine, National Taiwan University, No 1 Chang-Te Street, Taipei 10048, Taiwan. ${ }^{8}$ Tissue Regeneration Product Technology Division, Biomedical Technology and Device Research Laboratories, Industrial Technology Research Institute, Hsinchu County 310, Taiwan. ${ }^{9}$ Industrial Technology Research Institute, Rm. 635, Bldg. 53, No. 195, Sec. 4, Chung Hsing Rd, Chutung, Hsinchu, Taiwan.

Received: 3 May 2017 Accepted: 19 July 2017

Published online: 25 July 2017

\section{References}

1. Gustilo RB, Merkow RL, Templeman D. The management of open fractures. J Bone Joint Surg Am. 1990;72(2):299-304.

2. Walter G, Kemmerer M, Kappler C, Hoffmann R. Treatment algorithms for chronic osteomyelitis. Dtsch Arztebl Int. 2012;109(14):257-64.

3. Lew DP, Waldvogel FA. Osteomyelitis. Lancet. 2004;364(9431):369-79.

4. Costerton JW. Biofilm theory can guide the treatment of device-related orthopaedic infections. Clin Orthop Relat Res. 2005;437:7-11.

5. Marais LC, Ferreira N, Aldous C, Le Roux TL. The outcome of treatment of chronic osteomyelitis according to an integrated approach. Strategies Trauma Limb Reconstr. 2016;11(2):135-42.

6. Shen $Y$, Zhu W, Chen C, Nie Y, Lin X. Biofilm formation in attached microalgal reactors. Bioprocess Biosyst Eng. 2016;39(8):1281-8.

7. Wang J, Pantopoulos K. Regulation of cellular iron metabolism. Biochem J. 2011;:434(3):365-81.

8. Aisen P, Listowsky I. Iron transport and storage proteins. Annu Rev Biochem. 1980;49:357-93.

9. Watt RK. The many faces of the octahedral ferritin protein. Biometals. 2011;24(3):489-500.

10. Wang $Y X$, Hussain SM, Krestin GP. Superparamagnetic iron oxide contrast agents: physicochemical characteristics and applications in MR imaging. Eur Radiol. 2001;11(11):2319-31.

11. Lodhia J, Mandarano G, Nj F, Eu P, Cowell S. Development and use of iron oxide nanoparticles (part 1): synthesis of iron oxide nanoparticles for MRI. Biomed Imaging Interv J. 2010;6(2):e12.

12. Dutz S, Hergt R. Magnetic nanoparticle heating and heat transfer on a microscale: basic principles, realities and physical limitations of hyperthermia for tumour therapy. Int J Hyperthermia. 2013;29(8):790-800.

13. Yoshida $Y$, Urabe $K$, Furue $M$, Nakayama J. A case of cutaneous mycobacterium chelonae infection successfully treated with a combination of minocycline hydrochloride and thermotherapy. J Dermatol. 2004;31 (2):151-3.

14. Douple EB, Srohbehn JW, Bowers ED, Walsh JE. Cancer therapy with localized hyperthermia using an invasive microwave system. J Microw Power. 1979;14(2):181-6

15. Strohbehn JW, Bowers ED, Walsh JE, Douple EB. An invasive microwave antenna for locally-induced hyperthermia for cancer therapy. J Microw Power. 1979;14(4):339-50

16. Brezovich IA, Young JH. Hyperthermia with implanted electrodes. Med Phys. 1981;8(1):79-84.

17. Yu PK, Miller J, Cringle SJ, Yu DY. Experimental retinal ablation using a fourth-harmonic $266 \mathrm{~nm}$ laser coupled with an optical fiber probe. Invest Ophthalmol Vis Sci. 2006r;47(4):1587-93.

18. Robins HI, Rushing D, Kutz M, Tutsch $K D$, Tiggelaar CL, Paul D, Spriggs $D$, Kraemer C, Gillis W, Feierabend C, Arzoomanian RZ, Longo W, Alberti D, d'Oleire F, Qu RP, Wilding G, Stewart JA. Phase I clinical trial of melphalan 
and 41.8 degrees $C$ whole-body hyperthermia in cancer patients. J Clin Oncol. 1997;15(1):158-64.

19. Robins HI, Grosen E, Katschinski DM, Longo W, Tiggelaar CL, Kutz M, Winawer J, Graziano F. Whole body hyperthermia induction of soluble tumor necrosis factor receptors: implications for rheumatoid diseases. J Rheumatol. 1999:26(12):2513-6.

20. Gilchrist RK, Medal R, Shorey WD, Hanselman RC, Parrott JC, Taylor CB. Selective inductive heating of lymph nodes. Ann Surg. 1957;146(4):596-606.

21. Johannsen M, Gneveckow U, Thiesen B, Taymoorian K, Cho CH, Waldöfner N, Scholz R, Jordan A, Loening SA, Wust P. Thermotherapy of prostate cancer using magnetic nanoparticles: feasibility, imaging, and three-dimensional temperature distribution. Eur Urol. 2007;52(6):1653-61.

22. Johannsen M, Gneveckow U, Taymoorian K, Thiesen B, Waldöfner N, Scholz R, Jung K, Jordan A, Wust P, Loening SA. Morbidity and quality of life during thermotherapy using magnetic nanoparticles in locally recurrent prostate cancer: results of a prospective phase I trial. Int J Hyperthermia. 2007:23(3):315-23.

23. Johannsen M, Thiesen B, Wust P, Jordan A. Magnetic nanoparticle hyperthermia for prostate cancer. Int J Hyperthermia. 2010;26(8):790-5.

24. Al-Hezaimi K, Ramalingam S, Al-Askar M, ArRejaie AS, Nooh N, Jawad F, Aldahmash A, Atteya M, Wang CY. Real-time-guided bone regeneration around standardized critical size calvarial defects using bone marrowderived mesenchymal stem cells and collagen membrane with and without using tricalcium phosphate: an in vivo micro-computed tomographic and histologic experiment in rats. Int J Oral Sci. 2016;8:7-15.

25. Gao Y, Hu C, Zheng WJ, Yang S, Li F, Sun SD, Zrínyi M, Osada Y, Yang ZM Chen $\mathrm{YM}_{\mathrm{M}} \mathrm{Fe}_{3} \mathrm{O}_{4}$ anisotropic nanostructures in Hydrogels: efficient catalysts for the rapid removal of organic dyes from wastewater. ChemPhysChem. 2016;17(13):1999-2007.

26. Hall BB, Fitzgerald RH Jr, Rosenblatt JE. Anaerobic osteomyelitis. J Bone Joint Surg Am. 1983;65(1):30-5.

27. Haas DW, McAndrew MP. Bacterial osteomyelitis in adults: evolving considerations in diagnosis and treatment. Am J Med. 1996;101(5):550-61.

28. Calhoun JH, Manring MM. Adult osteomyelitis. Infect Dis Clin North Am. 2005;19(4):765-86.

29. Cierny $\mathrm{G}$ 3rd, Mader JT, Penninck JJ. A clinical staging system for adult osteomyelitis. Clin Orthop Relat Res. 2003:414(9):7-24.

30. Moroz P, Jones SK, Gray BN. Magnetically mediated hyperthermia: current status and future directions. Int J Hyperthermia. 2002;18(4):267-84.

31. Minamimura T, Sato H, Kasaoka S, Saito T, Ishizawa S, Takemori S, Tazawa K, Tsukada K. Tumor regression by inductive hyperthermia combined with hepatic embolization using dextran magnetite-incorporated microspheres in rats. Int J Oncol. 2000; 16(6):1153-1158.

32. Gupta AK, Gupta M. Synthesis and surface engineering of iron oxide nanoparticles for biomedical applications. Biomaterials. 2005;26(18):3995-4021.

33. Ramaswamy B, Kulkarni SD, Villar PS, Smith RS, Eberly C, Araneda RC, Depireux DA, Shapiro B. Movement of magnetic nanoparticles in brain tissue: mechanisms and impact on normal neuronal function. Nanomedicine. 2015;11(7):1821-9.

34. Cho $\mathrm{H}$, de Jong WA, Soderquist CZ. Probing the oxygen environment in $\mathrm{UO}(2)(2+)$ by solid-state 170 nuclear magnetic resonance spectroscopy and relativistic density functional calculations. J Chem Phys. 2010;132(8):084501.

35. Mahendran V, Philip J. Sensing of biologically important cations such as $\mathrm{Na}(+), \mathrm{K}(+), \mathrm{Ca}(2+), \mathrm{Cu}(2+)$, and $\mathrm{Fe}(3+)$ using magnetic nanoemulsions. Langmuir. 2013;29(13):4252-8.

36. Elliott DW, Zhang WX. Field assessment of nanoscale bimetallic particles for groundwater treatment. Environ Sci Technol. 2001;35(24):4922-6.

37. Lahonian M, Golneshan AA. Numerical study of temperature distribution in a spherical tissue in magnetic fluid hyperthermia using lattice Boltzmann method. IEEE Trans Nanobioscience. 2011;10(4):262-8.

38. Golneshan AA, Lahonian M. The effect of magnetic nanoparticle dispersion on temperature distribution in a spherical tissue in magnetic fluid hyperthermia using the lattice Boltzmann method. Int J Hyperthermia. 2011;27(3):266-74.

39. Lewis JA, Miller DR, Davies SG. Osteomyelitis complicating three types of traumatic hand wound. J Wound Care. 2004;13(7):281-3.

40. Eerenberg JP, Patka P, Haarman HJ, Dwars BJ. A new model for posttraumatic osteomyelitis in rabbits. J Invest Surg. 1994;7(5):453-65.

41. Lindsey BA, Clovis NB, Smith ES, Salihu S, Hubbard DF. An animal model for open femur fracture and osteomyelitis: part I. J Orthop Res. 2010;28(1):38-42.
42. Lindsey BA, Clovis NB, Smith ES, Salihu S, Hubbard DF. An animal model for open femur fracture and osteomyelitis-part II: Immunomodulation with systemic IL-12. J Orthop Res. 2010;28(1):43-7.

43. Subasi M, Kapukaya A, Kesemenli C, Kaya H, Sari I. Effect of granulocytemacrophage colony-stimulating factor on treatment of acute osteomyelitis. An experimental investigation in rats. Arch Orthop Trauma Surg. 2001;121(3):170-3.

44. Chai H, Guo L, Wang X, Fu Y, Guan J, Tan L, Ren L, Yang K. Antibacterial effect of 317L stainless steel contained copper in prevention of implant-related infection in vitro and in vivo. J Mater Sci Mater Med. 2011;22(11):2525-35.

45. Ozturan KE, Yucel I, Kocoglu E, Cakici H, Guven M. Efficacy of moxifloxacin compared to teicoplanin in the treatment of implant-related chronic osteomyelitis in rats. J Orthop Res. 2010t;28(10):1368-72.

46. Donlan RM, Costerton JW. Biofilms: survival mechanisms of clinically relevant microorganisms. Clin Microbiol Rev. 2002;15(2):167-93.

47. Parsek MR, Singh PK. Bacterial biofilms: an emerging link to disease pathogenesis. Annu Rev Microbiol. 2003;57:677-701.

48. Jordan A, Scholz R, Maier-Hauff K, van Landeghem FK, Waldoefner N, Teichgraeber U, Pinkernelle J, Bruhn H, Neumann F, Thiesen B, von Deimling A Felix R. The effect of thermotherapy using magnetic nanoparticles on rat malignant glioma. J Neurooncol. 2006;78(1):7-14.

49. Maier-Hauff K, Rothe R, Scholz R, Gneveckow U, Wust P, Thiesen B, Feussner A, von Deimling A, Waldoefner N, Felix R, Jordan A. Intracranial thermotherapy using magnetic nanoparticles combined with external beam radiotherapy: results of a feasibility study on patients with glioblastoma multiforme. J Neurooncol. 2007;81(1):53-60.

\section{Submit your next manuscript to BioMed Central and we will help you at every step:}

- We accept pre-submission inquiries

- Our selector tool helps you to find the most relevant journal

- We provide round the clock customer support

- Convenient online submission

- Thorough peer review

- Inclusion in PubMed and all major indexing services

- Maximum visibility for your research

Submit your manuscript at www.biomedcentral.com/submit
Biomed Central 